\title{
Curcumin improves diabetes mellitus-associated cerebral infarction by increasing the expression of GLUT1 and GLUT3
}

\author{
MINGYU XIA ${ }^{1 *}$, ZANKAI YE $^{2 *}$, YANFENG SHI $^{3}$, LIBO ZHOU $^{4}$ and YANG HUA \\ ${ }^{1}$ Vascular Ultrasonography Department, Xuanwu Hospital, Capital Medical University, Beijing 100053; \\ ${ }^{2}$ Structural Heart Disease Department, Fuwai Hospital, Chinese Academy of Medical Sciences, Beijing 100037; \\ ${ }^{3}$ Radiology Department, Daqing Longnan Hospital, Daqing, Heilongjiang 163453; ${ }^{4}$ Traditional Chinese Medicine Department, \\ Guang'anmen Hospital, China Academy of Chinese Medical Sciences, Beijing 100053, P.R. China
}

Received May 7, 2017; Accepted October 3, 2017

DOI: $10.3892 / \mathrm{mmr} .2017 .8085$

\begin{abstract}
Curcumin is characterized by anti-inflammatory, anti-oxidative, antiviral, antifibrotic, anticoagulation and glucose regulatory functions. However, whether it is protective in diabetes mellitus-associated cerebral infarction remains to be fully elucidated. In the present study, it was demonstrated for the first time, to the best of our knowledge, that curcumin markedly improved neurological deficits, cerebral infarct volume and brain edema rate following middle cerebral artery occlusion (MCAO) surgery. It was also shown that the expression levels of glucose transporter (GLUT)1 and GLUT3 were reduced in the MCAO group. However, following curcumin treatment, the levels of GLUT1 and GLUT3 were markedly increased. In addition, curcumin markedly decreased cell apoptosis, indicating an anti-apoptotic role of curcumin in the brain. To further evaluate whether curcumin prevented cell apoptosis by modulating the expression of GLUT1 and GLUT3, small interfering RNAs targeting GLUT1 and GLUT3 were selected. It was found that the knockdown of GLUT1 and GLUT3 inhibited the abundance of GLUT1, GLUT3 and B-cell lymphoma 2, even following incubation with curcumin. These data showed that curcumin protected brain cells from apoptosis and cerebral infarction, predominantly by upregulating GLUT1 and GLUT3.
\end{abstract}

\section{Introduction}

With improved living conditions, dietary changes and reduced labor intensity, the incidence of diabetes mellitus is rapidly

Correspondence to: Dr Yang Hua, Vascular Ultrasonography Department, Xuanwu Hospital, Capital Medical University, 45 Changchun Road, Xicheng, Beijing 100053, P.R. China E-mail: ag20170409@126.com

\section{*Contributed equally}

Key words: curcumin, cerebral infarction, glucose transporter 1, glucose transporter 3 increasing worldwide (1-3). Diabetic patients with poor glucose control can experience multiple complications, including cardiovascular disease, kidney disease, blindness and amputation, which not only seriously affects the quality of life of patients, but also imposes a heavy burden on family and society (4-6). Cerebral infarction is one of the major complications and one of the main causes of mortality associated with diabetes (5). Previous studies have shown that diabetes is one of the independent risk factors of stroke; diabetic patients succumb to cerebrovascular complication-associated mortality more frequently, compared with non-diabetic patients $(3,6,7)$. Therefore, the prevention and treatment of diabetes complicated by cerebral infarction requires investigation to reduce the mortality and disability rates of patients.

Under physiological conditions, the brain is unable to store carbohydrates, so the maintenance of brain function is almost entirely dependent on the constant supply of glucose from the blood for the production of ATP $(8,9)$. Therefore, the transport of glucose into the brain is a key step in maintaining brain metabolism, which is regulated by a glucose transporter (GLUT) (10). At present, 13 types of GLUT proteins have been identified in mammalian cells $(11,12)$. The transporters responsible for glucose transport in the brain are predominantly GLUT1 and GLUT3 (13). Investigations have shown that GLUT1 is divided into two types: $55 \mathrm{kDa}$ GLUT1 is located in the blood-brain barrier (BBB), whereas $45 \mathrm{kDa}$ GLUT1 is present in the choroid plexus ependymal epithelial cells and glial cells, which are mainly responsible for glucose transport through the BBB to the brain $(14,15)$. GLUT3 is predominantly expressed in neurons, and is rich in neuronal dendrites and axons. This is possibly due to dendritic and axonal cells lacking high levels of mitochondria and the anaerobic glycolysis of glucose being insufficient $(16,17)$. A continuous supply of glucose is necessary to ensure brain bioelectrical activity. Therefore, investigating the dynamic changes in the expression of GLUT1 and GLUT3 in the brain is of value for the clinical treatment of diabetes.

Jiang Huang (Curcuma longa L) is also known as turmeric, which originates from China, Japan and the United States $(18,19)$. Jiang Huang has been approved by the U.S. Food and Drug Administration as a natural food additive (20). Curcumin is extracted from turmeric as a soluble phenolic 
pigment, which is widely used in food additives $(21,22)$. Multiple studies have shown that curcumin has several pharmacological effects, including anti-inflammatory, anti-oxidative, antiviral, antifibrotic and anticoagulation effects, and lipid regulation $(21,22)$. Due to its low side effects and its safety, curcumin offers promising potential for clinical applications and has received increasing attention in investigations.

In the present study, it was demonstrated for the first time, to the best of our knowledge, that curcumin significantly improved diabetes mellitus-associated cerebral infarction, primarily by increasing the expression of GLUT1 and GLUT3.

\section{Materials and methods}

Establishment of a diabetic rat model. Adult male Sprague-Dawley rats (220-230 g), provided by the Animal Facility of the Health Science Center of Peking University (Beijing, China) were housed in the laboratory animal room and maintained at $25 \pm 1^{\circ} \mathrm{C}$ with $65 \pm 5 \%$ humidity on a 12 -h light/dark cycle (lights on between 07:30 a.m. and 19:30 p.m.) for at least 1 week prior to the experiments. The animals were provided with food and water ad libitum. The rats were fed for 7 days and fasted for $12 \mathrm{~h}$. In the experimental group, streptozotocin dissolved in citric acid sodium citrate buffer ( $\mathrm{pH} 4.5$ ) was injected into the left lower abdominal cavity at a dose of $60 \mathrm{mg} / \mathrm{kg}$. The control group was injected with the same dose of citrate buffer. At $72 \mathrm{~h}$ post-injection, blood glucose was measured in the tail vein blood of the rats (One-Touch Johnson Ultra glucose meter). A blood glucose level $>16.7 \mathrm{mmol} / \mathrm{l}$ was defined as diabetic. Blood glucose and body weights were measured once each week.

All experimental protocols described in the present study were approved by the Ethics Review Committee for Animal Experimentation of Xuanwu Hospital Capital Medical University (Beijing, China).

Transient middle cerebral artery occlusion (MCAO). The rats were subjected to transient focal cerebral ischemia induced by right MCAO with modifications. In brief, the rats were anesthetized with $10 \%$ chloral hydrate $(360 \mathrm{mg} / \mathrm{kg}$; i.p.), following which arterial blood samples were obtained via a femoral catheter, and measurements of $\mathrm{pO}_{2}, \mathrm{pCO}_{2}$ and $\mathrm{pH}$ were recorded using an AVL 998 Blood Gas Analyzer (Roche Diagnostics, Basel, Switzerland). Rectal temperature was maintained at $37 \pm 0.5^{\circ} \mathrm{C}$ during $\mathrm{MCAO}$ via a temperature-regulated heating lamp. A fiber-optic probe was attached to the parietal bone overlying the MCA territory 5-mm posterior and 5-mm lateral to the bregma, and was connected to a laser-Doppler flowmeter (Perifluxsystem 5000; Perimend, Stockholm, Sweden) for continuous monitoring of the cerebral blood flow (CBF). A 40 nylon monofilament suture with a heat-blunted tip was introduced into the internal carotid artery through the stump of the external carotid artery, gently advanced for a distance of $18 \mathrm{~mm}$ from the common carotid artery bifurcation to block the origin of the MCA for $90 \mathrm{~min}$, and then withdrawn to allow reperfusion. Only animals, which exhibited a reduction in $\mathrm{CBF}>85 \%$ during right $\mathrm{MCAO}$ and a $\mathrm{CBF}$ recovery $>80 \%$ following $10 \mathrm{~min}$ of reperfusion were included in the study. Following close of the wounds, the animals were allowed to recover from anesthesia prior to being returned to their housing cages. For the curcumin treatment group, the rats were administered with curcumin by gavage at a dose of $40 \mathrm{mg} / \mathrm{kg}$, and saline was used as a control (Con).

Assessment of neurological deficit scores and analysis of survival rates. The neurological deficit scores were assessed prior to sacrifice of the rats $24 \mathrm{~h}$ following reperfusion as described previously $(23,24)$. Each rat was assessed and scored by two examiners who remained blinded to the identity of the rat and the treatment protocol. The following neurological deficit scoring system was used: 0 , no motor deficits (normal); 1 , forelimb weakness and torso turning to the ipsilateral side when held by tail (mild); 2, circling to the contralateral side but normal posture at rest (moderate); 3, unable to bear weight on the affected side at rest (severe); and 4, no spontaneous locomotor activity or barrel rolling (critical). If no deficit was observed $2 \mathrm{~h}$ following recovering from anesthesia, the animal was removed from further experiments.

Edema measurement. The rats were sacrificed by decapitation under deep anesthesia with $10 \%$ chloral hydrate $(360 \mathrm{mg} / \mathrm{kg}$, i.p.) at $6,12,24$ or $72 \mathrm{~h}$ following reperfusion. The ipsilateral and contralateral hemispheres were dissected, and the wet weight of the tissue was determined. The tissues were dried at $120^{\circ} \mathrm{C}$ for $24 \mathrm{~h}$. The percentage of cerebral water was determined as follows: (wet weight-dry weight)/dry weight x100.

Measurement of infarct volume. Following reperfusion, the rats were deeply anesthetized with $10 \%$ chloral hydrate and then sacrificed; following which the whole brain was rapidly removed. Coronal sections ( $n=10$ for each group) were cut into 2-mm sections and stained with standard 2\% 2,3,5-triphenyltetrazolium chloride (TTC; Sigma-Aldrich; Merck Millipore, Darmstadt, Germany) for $10 \mathrm{~min}$ at $37^{\circ} \mathrm{C}$, followed by overnight immersion in $4 \%$ formalin. Infarct volume, expressed as a percentage of whole brain volume, was measured using an image processing and analysis system (1.25X objective; Q570IW; Leica Microsystems GmbH, Wetzlar, Germany) and was calculated by integration of the infarct area on each brain section along the rostral-caudal axis.

Hematoxylin and eosin $(H \& E)$ staining. The rats were sacrificed 24 and $72 \mathrm{~h}$ post-MCAO with an overdose of $10 \%$ chloral hydrate $(360 \mathrm{mg} / \mathrm{kg}$, i.p.) and were transcardially perfused with $0.9 \%$ saline solution followed by $4 \%$ ice-cold phosphate-buffered paraformaldehyde. The brains were removed, fixed overnight, and equilibrated in phosphate-buffered $30 \%$ sucrose. Coronal sections of 1.0-2.0 $\mathrm{mm}$ from the bregma were cut on a cryostat (Leica CM3,000; Leica Microsystems $\mathrm{GmbH})$ to a thickness of $25-\mu \mathrm{m}$ and used for H\&E staining under an inverted microscope (XDS-500D; Shanghai Caikon Optical Instrument Co., Ltd., Shanghai, China).

Transmission electron microscopy (TEM). TEM was used to evaluate the ultrastructural changes of brain sections. Cerebral fragments were fixed with $2.5 \%$ glutaraldehyde solution overnight at $4^{\circ} \mathrm{C}$; following which they were washed with PBS and fixed with $1 \%$ osmic acid at $4^{\circ} \mathrm{C}$ for $2 \mathrm{~h}$. The tissues were embedded in an epon/araldite mixture. Ultra-thin sections were cut at $90 \mathrm{~nm}$ and stained with uranyl acetate and lead 
citrate. The samples were observed under a 1230 type TEM (JEOL, Ltd., Tokyo, Japan) and images were captured.

TUNEL staining. Nuclear fragmentation was detected using TUNEL staining with an apoptosis detection kit (R\&D Systems, Inc., Minneapolis, MN, USA) according to the manufacturer's protocol.

Protein extraction, western blot analysis and antibodies. Cellular proteins were extracted using RIPA buffer containing $50 \mathrm{mM}$ Tris/ $\mathrm{HCl}$ (pH 7.4), $150 \mathrm{mM} \mathrm{NaCl}, 1 \%$ (v/v) NP-40 and $0.1 \%(\mathrm{w} / \mathrm{v})$ SDS with $1 \%$ (v/v) PMSF (Solarbio Science \& Technology Co., Ltd. Beijing, China), $0.3 \%$ (v/v) protease inhibitor (Sigma; Merck Millipore) and $0.1 \%$ (v/v) phosphorylated proteinase inhibitor (Sigma; Merck Millipore). The lysates were centrifuged at $10,000 \mathrm{x}$ g at $4^{\circ} \mathrm{C}$ for $15 \mathrm{~min}$ and the supernatant was collected for total protein. A BCA protein assay kit (Pierce; Thermo Fisher Scientific, Inc.) was used to determine the protein concentration. Equal quantities of protein $(15 \mu \mathrm{g})$ were separated on an SDS-PAGE gel $(10 \%$ $\mathrm{v} / \mathrm{v}$ ) polyacrylamide) and transferred onto a PVDF membrane. Nonspecific binding was blocked using $8 \%$ (w/v) milk in TBS-T for $2 \mathrm{~h}$ at room temperature. The membranes were then incubated with primary antibodies against $\beta$-actin (cat. no. 4970; 1:1,000; Cell Signaling Technology, Inc., Boston, MA, USA), GLUT1 (cat. no. 12939; 1:1,000; Cell Signaling Technology, Inc.), GLUT3 (cat. no. ab41525; 1:1,000; Abcam, Cambridge, UK), B-cell lymphoma 2 (Bcl-2; cat. no. ab59348; 1:1,000; Abcam), and Bcl-2-associated X protein (Bax; cat. no. ab32503; 1:1,000, Abcam) overnight at $4^{\circ} \mathrm{C}$. Following several washes with TBST, the membranes were incubated with HRP-conjugated goat anti-rabbit IgG (cat. no. ZB-2306; 1:5,000; OriGene Technologies, Inc., Beijing, China) for $2 \mathrm{~h}$ at room temperature and then washed. The target proteins were visualized using enhanced chemiluminescence (Merck KGaA, Darmstadt, Germany) according to the manufacturer's recommendations, quantified using density analysis normalized against $\beta$-actin, according to the manufacturer's protocol (ImageJ version 1.8.0, National Institutes of Health, Bethesda, MD, USA), and expressed as the fold-change, compared with the control.

N2 a cell culture. The N2a cell line was obtained from Professor Huaxi Xu (Biomedical Research, Xiamen University, Xiamen, China). Briefly, the cells were grown in DMEM with $10 \%$ FBS (Invitrogen; Thermo Fisher Scientific, Inc.) and 1\% penicillin/streptomycin and incubated at $37^{\circ} \mathrm{C}$ with $5 \% \mathrm{CO}_{2} / 95 \%$ air in a humidified atmosphere.

Cell transfection. The small interfering (si)RNAs targeting GLUT1 or GLUT3 or a non-specific siRNA (NC) were purchased from Genepharma Co., Ltd. (Shanghai, China). Transfection of si-GLUT1 (sense, 5'-GGAATTCAATGCT GATGATGA-3' and antisense 5'-TCATCATCAGCATTG AATTCC-3'), si-GLUT3 (sense 5'-CTACCTGTCAACAGC GTTTC-3' and antisense 5'-CAGTATGTGACCAATGTAC-3') or NC (sense 5'-TTCTCCGAACGTGTCACGT-3' and antisense 5'-ACGTGACACGTTCGGAGAA-3') was performed with HiperFect transfection reagent (Qiagen, Inc., Valencia, CA, USA) according to the manufacturer's protocol. In brief,
$6 \times 10^{5}$ cells were equally seeded in 6-well plates with $2 \mathrm{ml}$ DMEM containing serum and antibiotics. At the same time, si-GLUT1, si-GLUT3 or NC were mixed with HiperFect transfection reagent (Qiagen, Inc.) and incubated at room temperature for $10 \mathrm{~min}$. The complex was then respectively transfected into the cells for $48 \mathrm{~h}$.

Statistical analysis. All data are presented as the mean \pm standard deviation. Statistical significance was analyzed using one-way analysis of variance followed by Tukey's test for multiple comparisons. Statistical analysis was carried out with Student's t-test (GraphPad Prism 7; GraphPad Software, Inc., La Jolla, CA, USA). The two-tailed unpaired Student's t-test was used for comparing the band density values between groups. $\mathrm{P}<0.05$ was considered to indicate a statistically significant difference.

\section{Results}

Curcumin improves cerebral infarction in rats. Cerebral infarction was examined using TTC staining. As shown in Fig. 1A, MCAO induced cerebral infarction. MCAO treatment also significantly induced neurological deficits, cerebral infarct volume and brain edema rate, whereas curcumin markedly improved neurological deficits, cerebral infarct volume and brain edema rate (Fig. 1B-D). In addition, the neuron count was markedly reduced following MCAO treatment, whereas neuron count was enhanced following curcumin treatment (Fig. 1E). These data indicated the protective role of curcumin in cerebral infarction of diabetic rats.

TEM analysis indicates that curcumin treatment alleviates $I / R$ injury-induced neuron damage. To determine whether curcumin treatment induced the stabilization of neuronal and ultrastructure in the peri-infarct cortex, TEM was performed. Normal cortical neurons had large oval nuclei with a clear nuclear membrane and normal mitochondria (Fig. 2). In the I/R group, irregular nuclear membranes, chromatin condensation and numerous vacuoles were identified in the neurons (Fig. 2). By contrast, the above changes were alleviated in the curcumin group (Fig. 2). These results indicated that curcumin treatment alleviated neuron damage.

Curcumin enhances cerebral expression of GLUT1 and GLUT3. Subsequently, the present study examined the expression of GLUT1 and GLUT3 in the rat brains. Compared with the control group, reduced expression levels of GLUT1 and GLUT3 were shown in the MCAO group (Fig. 3A). However, following curcumin treatment, the levels of GLUT1 and GLUT3 were markedly increased (Fig. 3B).

Curcumin reduces cell apoptosis in rat brains. The present study also evaluated the apoptotic cells in the rat brains. Compared with the control group, MCAO treatment significantly increased the number of apoptotic cells (Fig. 4A and B). However, curcumin markedly reduced cell apoptosis (Fig. 4A and B). The results of the western blot analysis showed that curcumin increased the expression of $\mathrm{Bcl}-2$ but reduced the expression of Bax (Fig. 4C), indicating an anti-apoptotic role of curcumin in the cerebral brain. 
A

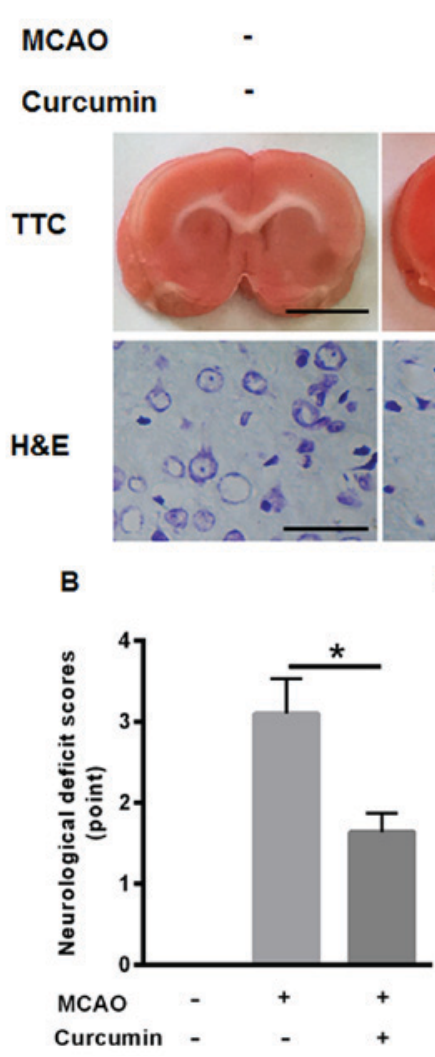

E

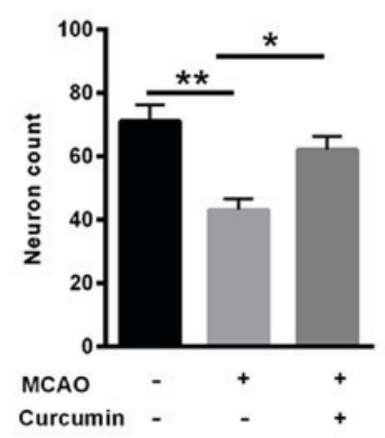

C
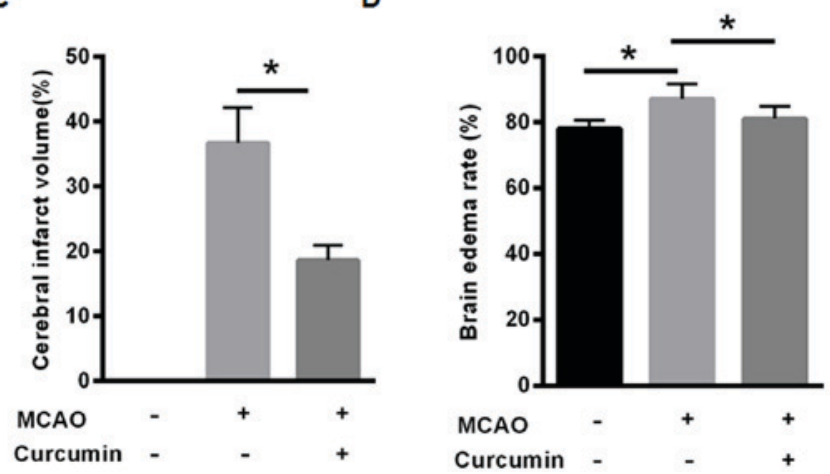

Figure 1. Curcumin improves cerebral infarction in rats. (A) TTC and H\&E staining. Compared with the MCAO group, curcumin markedly improved (B) neurological deficits, (C) cerebral infarct volume and (D) brain edema rate. (E) Neuron count was enhanced by curcumin treatment. ${ }^{*} \mathrm{P}<0.05$; ${ }^{* *} \mathrm{P}<0.01 \mathrm{TTC}$, 2,3,5-triphenyltetrazolium chloride; H\&E, hematoxylin and eosin; MCAO, middle cerebral artery occlusion.
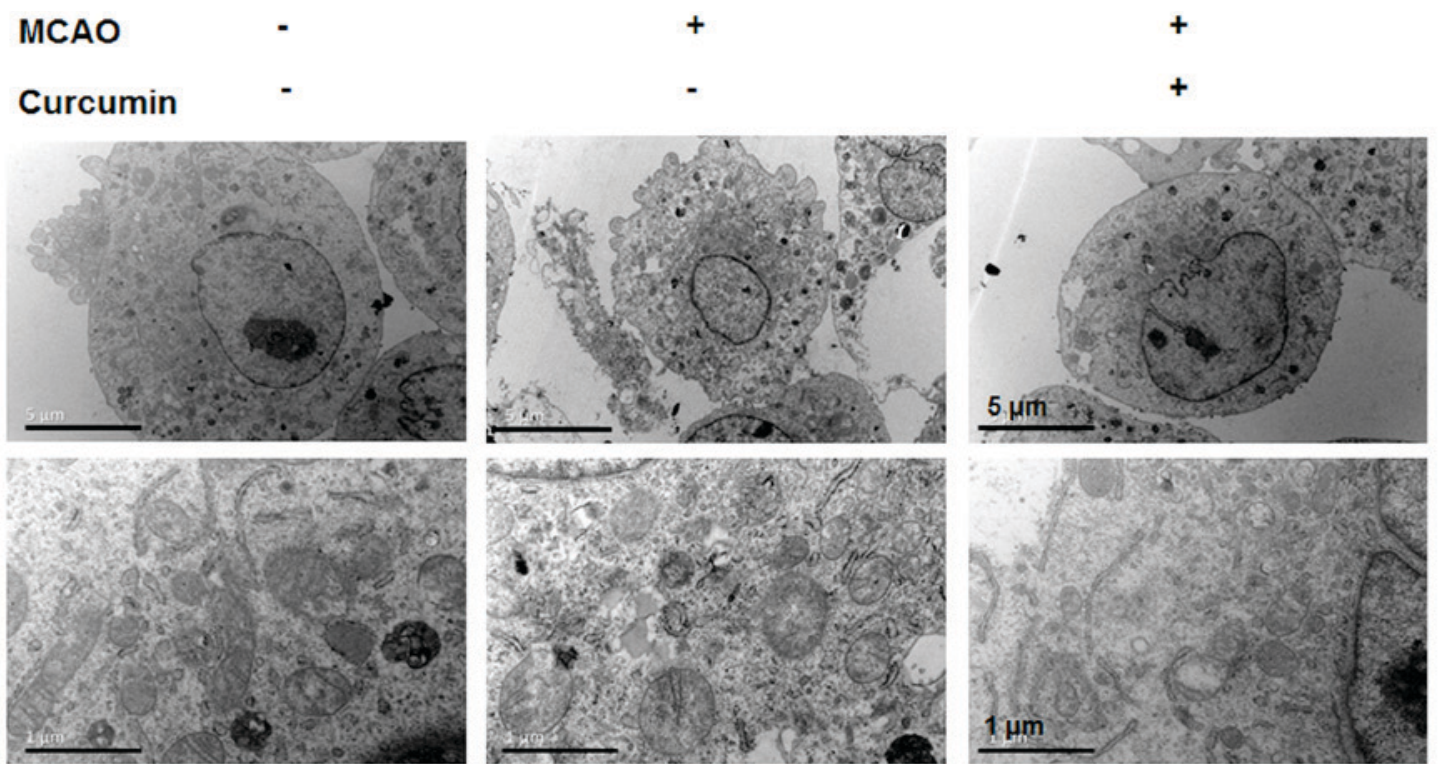

Figure 2. Transmission electron microscope analysis indicated that curcumin treatment alleviated ischemia/reperfusion injury-induced neuron damage.

Curcumin protects brain cells from apoptosis mainly by upregulating GLUT1 and GLUT3. To further evaluate whether curcumin protected against cell apoptosis by modulating the expression of GLUT1 and GLUT3, siRNAs targeting GLUT1 and GLUT3 were selected and used to transfect cells. As shown in Fig. 5A, curcumin treatment enhanced the expression of
GLUT1. The siRNA targeting GLUT1 significantly suppressed the level of GLUT1 and Bcl-2, even following curcumin treatment (Fig. 5A). Similarly, the expression of GLUT3 was increased in the cells treated with curcumin (Fig. 5B), whereas silencing GLUT3 inhibited the levels of GLUT3 and Bcl-2, even following curcumin incubation (Fig. 5B). These data 
A

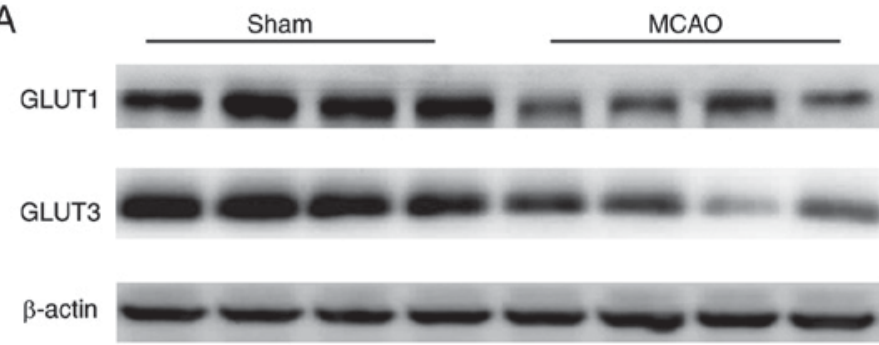

B

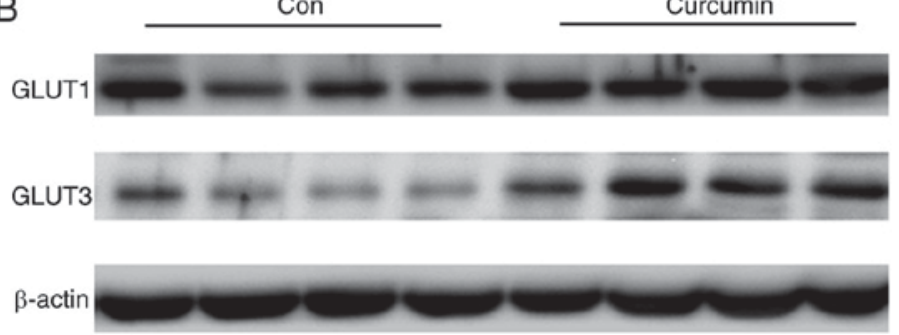

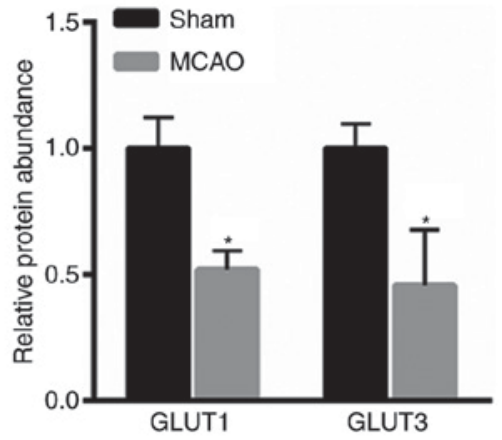

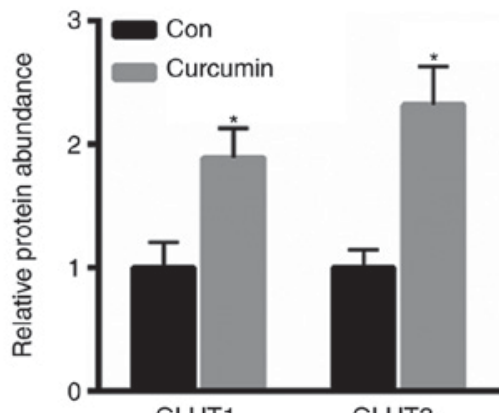

Figure 3. Curcumin enhances cerebral expression of GLUT1 and GLUT3. (A) Compared with the control group, the expression levels of GLUT1 and GLUT3 were reduced in the MCAO group. (B) Curcumin treatment increased the levels of GLUT1 and GLUT3. "P $<0.05$, vs. Sham or Con. GLUT, glucose transporter; MCAO, middle cerebral artery occlusion; Con, control.

A

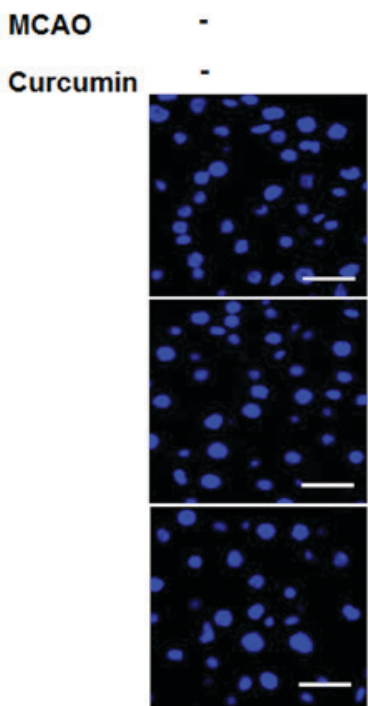

DAPI

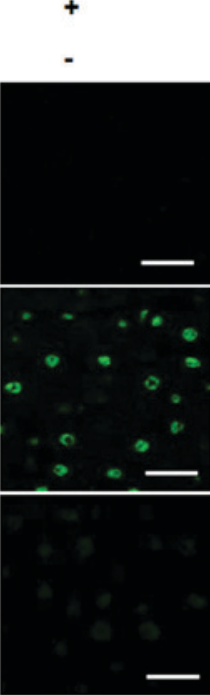

TUNEL
B

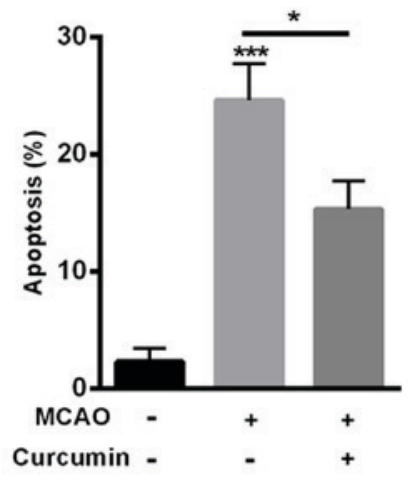

Merge
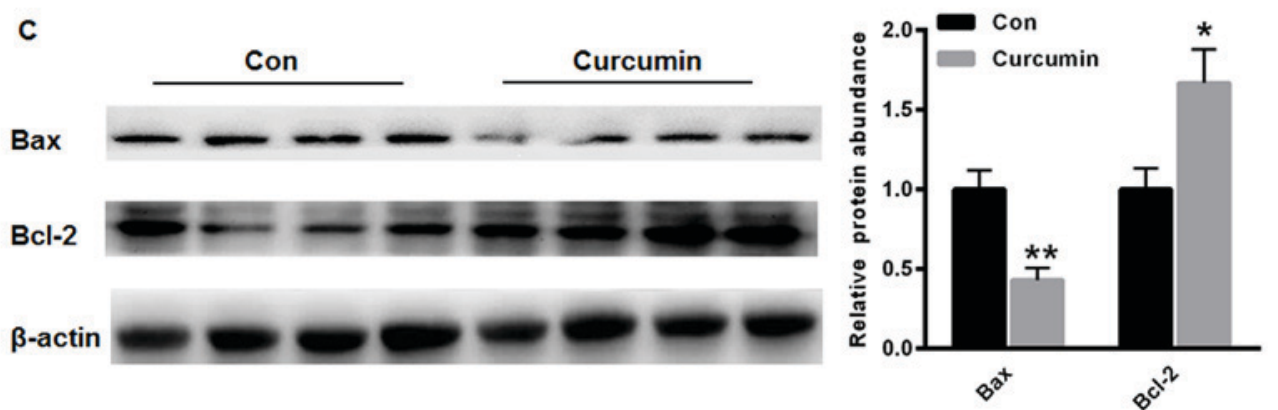

Figure 4. Curcumin reduces cell apoptosis in rat brains. (A) TUNEL staining. (B) Statistical analysis of apoptotic cells. (C) Western blot analysis showed that curcumin increased the expression of Bcl-2 but reduced the expression of Bax. ${ }^{*} \mathrm{P}<0.05,{ }^{* * *} \mathrm{P}<0.01,{ }^{* * * *} \mathrm{P}<0.001$, vs. control. Bcl-2, B-cell lymphoma 2; Bax, Bcl-2-associated X factor; MCAO, middle cerebral artery occlusion; Con, control. 
A

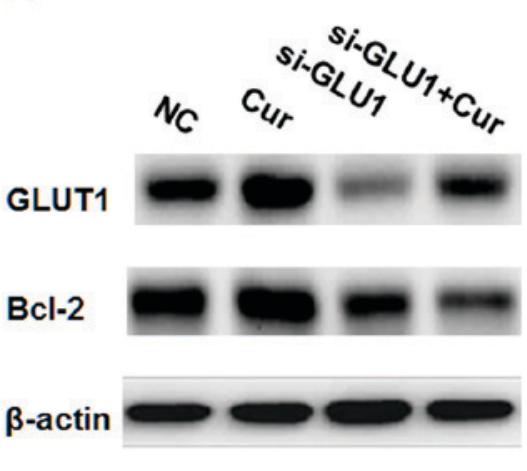

B

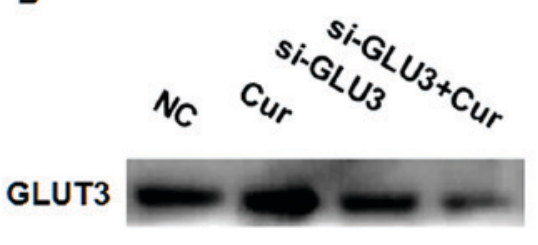

Bcl-2

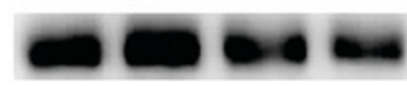

$\beta$-actin
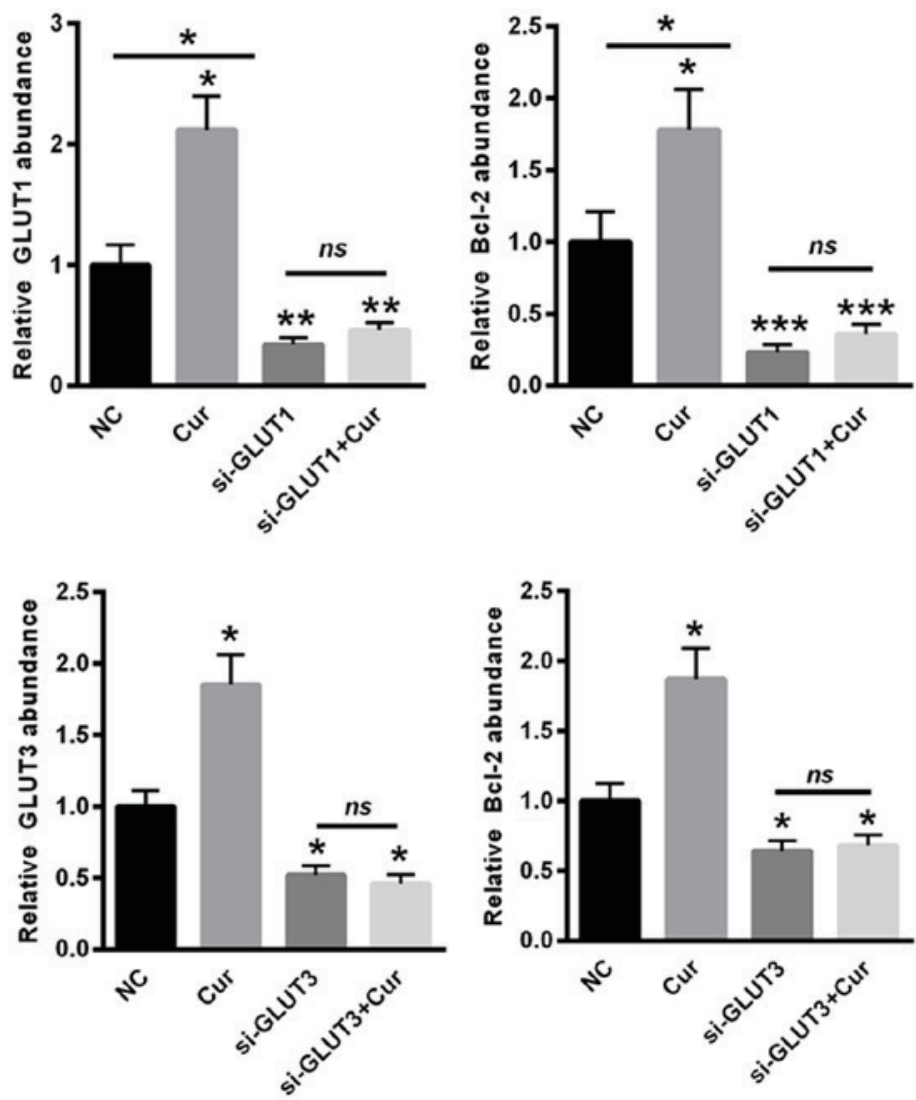

Figure 5. Curcumin protects brain cells from apoptosis mainly by upregulating GLUT1 and GLUT3. (A) siRNA targeting GLUT1 significantly suppressed the levels of GLUT1 and Bcl-2, even following curcumin treatment. (B) Knockdown of GLUT3 reduced the levels of GLUT3 and Bcl-2, even following curcumin incubation. ${ }^{*} \mathrm{P}<0.05,{ }^{* *} \mathrm{P}<0.01,{ }^{* * *} \mathrm{P}<0.001$ between the groups underlying the comparison lines. B-cell lymphoma 2; GLUT, glucose transporter; Cur, curcumin; si, small interfering RNA; NC, negative control; ns, not significant.

showed that curcumin protected the brain cells from apoptosis, predominantly by upregulating the expression of GLUT1 and GLUT3.

\section{Discussion}

The main risks of diabetes are chronic complications caused by chronic hyperglycemia, and some of the most important complications are vascular complications $(3,6)$. It is reported that $75 \%$ of diabetic patients worldwide succumbed to diabetic vascular complication-associate mortality, and this mortality rate ranks as the second highest of total causes of diabetes-associated mortality $(7,23)$. There are also regional differences in the mortality and disability rates of vascular complications. Diabetic patients with cerebrovascular disease have 2-4 times the mortality rate of non-diabetic patients (5). Diabetic cerebrovascular disease is mainly caused by diabetic cerebral infarction, which accounts for $85 \%$ of diabetic cerebrovascular disease $(8,25)$. The prevalence of the diabetic vascular disease rate increases with increasing duration of diabetes, ischemic lesions rather than hemorrhagic lesions, particularly in lacunar infarction, and due to repeated relapse of the disease, therefore, the prevention and cure of cerebral infarction is key to reducing diabetes morbidity rates $(5,6)$.

Curcumin is reported to significantly inhibit the growth, metastasis, invasion and colony formation of multiple tumors (26-28). In the present study, it was demonstrated that curcumin treatment in rats with diabetes improved cerebral infarction. The brain is almost entirely dependent on glucose for energy, and glucose is transported from the blood circulation into the brain cells through two main processes $(14,15)$. The first process is through the BBB by the GLUT1 transporter. The glucose is then transported into the cells, mainly through the cell membrane (17). In the condition of diabetes, chronic hyperglycemia can decrease glucose transport (15). As a protective mechanism, the downregulation of glucose intracellular flow reduces the cytotoxic effect of high glucose. However, a long-term reduction in glucose supply may cause a series of disadvantages. Multiple studies have shown that this downregulation is mediated by glucose transporters $(10,16)$. Therefore, identifying how to improve the expression of GLUT1 and GLUT3 glucose transporters in the presence of chronic hyperglycemia in diabetes is important.

In the present study, the data demonstrated that curcumin significantly enhanced the expression of GLUT1 and GLUT3, suggesting an improved glucose supply in the rat brains. The present study also evaluated whether curcumin reduced the cell apoptosis induced by cerebral infarction. TUNEL staining and western blot analysis showed that curcumin reduced the number of apoptotic cells and increased the expression of Bcl-2, indicating an anti-apoptotic role of curcumin in the cerebral brain. Of note, it was found that the siRNA targeting GLUT1 or GLUT3 significantly suppressed the levels of GLUT1, GLUT3 and Bcl-2, even following curcumin treatment. These 
data showed that curcumin protected the brain cells from apoptosis, mainly by upregulating GLUT1 and GLUT3.

In conclusion, the present study is the first, to the best of our knowledge, to demonstrate that curcumin improved diabetes-induced cerebral infarction, mainly by enhancing the expression of GLUT1 and GLUT3 in the rat brain.

\section{References}

1. Zhao L and Hu FX: $\alpha$-Lipoic acid treatment of aged type 2 diabetes mellitus complicated with acute cerebral infarction. Eur Rev Med Pharmacol Sci 18: 3715-3719, 2014.

2. Liu X, Zhou Y, Wang J, Liu X, Yang C, Li J, Chen S and Wu S: Effect of central obesity on the events of new-onset cerebral infarction among type 2 diabetes mellitus patients. Zhonghua Liu Xing Bing Xue Za Zhi 35: 390-392, 2014 (In Chinese).

3. Inagaki $K$, Nagao M and Oikawa S: Internal medicine and neurological diseases: Progress in diagnosis and treatment. Topics: II neurological diseases related to diabetes mellitus; 2 Cerebral infarction, coma, hypoglycemia. Nihon Naika Gakkai Zasshi 101: 2180-2187, 2012 (In Japanese).

4. Cambon H, Derouesne C, Yelnik A, Duyckaerts C and Hauw JJ: Effect of diabetes mellitus and blood glucose on the size of cerebral infarction and causes of death. Neuropathological study of 77 cases of infarction in the sylvian artery area. Rev Neurol (Paris) 147: 727-734, 1991

5. Du L, Ma J and Zhang X: Higher serum uric acid may contribute to cerebral infarction in patients with type 2 diabetes mellitus: A meta-analysis. J Mol Neurosci 61: 25-31, 2017.

6. Ichikawa H, Shimizu Y, Kuriki A, Murakami H, Mukai M and Kawamura M: The brainstem is at high risk for recurrent noncardioembolic cerebral infarction in association with diabetes mellitus: A hospital-based study. Eur Neurol 67: 26-32, 2012.

7. Kawamura T, Umemura T, Kanai A, Nagashima M, Nakamura N, Uno T, Nakayama M, Sano T, Hamada Y, Nakamura J and Hotta N: Soluble adhesion molecules and C-reactive protein in the progression of silent cerebral infarction in patients with type 2 diabetes mellitus. Metabolism 55: 461-466, 2006.

8. Long Y, Zhan Q, Yuan M, Duan X, Zhou J, Lu J, Li Z, Yu F, Zhou X, Yang Q and Xia J: The expression of microRNA-223 and FAM5C in cerebral infarction patients with diabetes mellitus. Cardiovasc Toxicol 17: 42-48, 2017.

9. Petzold S, Kapellen T, Siekmeyer M, Hirsch W, Bartelt H, Siekmeyer W and Kiess W: Acute cerebral infarction and extra pontine myelinolysis in children with new onset type 1 diabetes mellitus. Pediatr Diabetes 12: 513-517, 2011.

10. Tang HL, Li DD, Zhang JJ, Hsu YH, Wang TS, Zhai SD and Song YQ: Lack of evidence for a harmful effect of sodium-glucose co-transporter 2 (SGLT2) inhibitors on fracture risk among type 2 diabetes patients: A network and cumulative meta-analysis of randomized controlled trials. Diabetes Obes Metab 18: 1199-1206, 2016.

11. Mittal N, Mittal R, Kumar H and Medhi B: Sodium glucose co-transporter 2 inhibitors for glycemic control in type 2 diabetes mellitus: Quality of reporting of randomized controlled trials. Perspect Clin Res 7: 21-27, 2016.

12. Nunez DJ, Yao X, Lin J, Walker A, Zuo P, Webster L, Krug-Gourley S, Zamek-Gliszczynski MJ, Gillmor DS and Johnson SL: Glucose and lipid effects of the ileal apical sodium-dependent bile acid transporter inhibitor GSK2330672: Double-blind randomized trials with type 2 diabetes subjects taking metformin. Diabetes Obes Metab 18: 654-662, 2016.

13. Ohki T, Isogawa $\mathrm{A}$, Toda $\mathrm{N}$ and Tagawa $\mathrm{K}$ : Effectiveness of ipragliflozin, a sodium-glucose co-transporter 2 inhibitor, as a second-line treatment for non-alcoholic fatty liver disease patients with type 2 diabetes mellitus who do not respond to incretin-based therapies including glucagon-like peptide-1 analogs and dipeptidyl peptidase-4 inhibitors. Clin Drug Investig 36: 313-319, 2016.
14. Shin SJ, Chung S, Kim SJ, Lee EM, Yoo YH, Kim JW, Ahn YB, Kim ES, Moon SD, Kim MJ and Ko SH: Effect of sodium-glucose co-transporter 2 inhibitor, dapagliflozin, on renal renin-angiotensin system in an animal model of type 2 diabetes. PLoS One 11: e0165703, 2016.

15. Singh AK and Singh R: Sodium-glucose co-transporter-2 inhibitors and dipeptidyl peptidase- 4 inhibitors combination therapy in type 2 diabetes: A systematic review of current evidence. Indian J Endocrinol Metab 20: 245-253, 2016.

16. Tang H, Cui W, Li D, Wang T, Zhang J, Zhai S and Song Y: Sodium-glucose co-transporter 2 inhibitors in addition to insulin therapy for management of type 2 diabetes mellitus: A meta-analysis of randomized controlled trials. Diabetes Obes Metab 19: 142-147, 2017.

17. Yabe D, Iwasaki M, Kuwata H, Haraguchi T, Hamamoto $Y$, Kurose T, Sumita K, Yamazato H, Kanada S and Seino Y: Sodium-glucose co-transporter-2 inhibitor use and dietary carbohydrate intake in Japanese individuals with type 2 diabetes: A randomized, open-label, 3-arm parallel comparative, exploratory study. Diabetes Obes Metab 19: 739-743, 2017.

18. Aziz MT, El Ibrashy IN, Mikhailidis DP, Rezq AM, Wassef MA, Fouad HH, Ahmed HH, Sabry DA, Shawky HM and Hussein RE: Signaling mechanisms of a water soluble curcumin derivative in experimental type 1 diabetes with cardiomyopathy. Diabetol Metab Syndr 5: 13, 2013.

19. Margina D, Gradinaru D, Manda G, Neagoe I and Ilie M: Membranar effects exerted in vitro by polyphenols-quercetin, epigallocatechin gallate and curcumin-on HUVEC and Jurkat cells, relevant for diabetes mellitus. Food Chem Toxicol 61: 86-93, 2013.

20. Nishiyama T, Mae T, Kishida H, Tsukagawa M, Mimaki Y, Kuroda M, Sashida Y, Takahashi K, Kawada T, Nakagawa K and Kitahara M: Curcuminoids and sesquiterpenoids in turmeric (Curcuma longa L.) suppress an increase in blood glucose level in type 2 diabetic KK-Ay mice. J Agric Food Chem 53: 959-963, 2005.

21. Bulboacă A, D Bolboacă S and Suci S: Protective effect of curcumin in fructose-induced metabolic syndrome and in streptozotocin-induced diabetes in rats. Iran J Basic Med Sci 19: 585-593, 2016.

22. Castro CN, Barcala Tabarrozzi AE, Winnewisser J, Gimeno ML, Antunica Noguerol M, Liberman AC, Paz DA, Dewey RA and Perone MJ: Curcumin ameliorates autoimmune diabetes. Evidence in accelerated murine models of type 1 diabetes. Clin Exp Immunol 177: 149-160, 2014.

23. Li G, Xu X, Wang D, Wang J, Wang Y and Yu J: Microglial activation during acute cerebral infarction in the presence of diabetes mellitus. Neurol Sci 32: 1075-1079, 2011.

24. Zhang B, Wang D, Ji TF, Shi L and Yu JL: Overexpression of IncRNA ANRIL up-regulates VEGF expression and promotes angiogenesis of diabetes mellitus combined with cerebral infarction by activating NF- $\kappa$ B signaling pathway in a rat model. Oncotarget 8: 17347-17359, 2017.

25. Kobayashi S: Progress in diagnosis of and therapy for cerebral infarction in patients with diabetes mellitus. Nihon Naika Gakkai Zasshi 93: 1532-1538, 2004 (In Japanese).

26. Chiu J, Khan ZA, Farhangkhoee H and Chakrabarti S: Curcumin prevents diabetes-associated abnormalities in the kidneys by inhibiting p300 and nuclear factor-kappaB. Nutrition 25: 964-972, 2009.

27. Chuengsamarn S, Rattanamongkolgul S, Luechapudiporn R, Phisalaphong $\mathrm{C}$ and Jirawatnotai S: Curcumin extract for prevention of type 2 diabetes. Diabetes Care 35: 2121-2127, 2012.

28. El-Azab MF, Attia FM and El-Mowafy AM: Novel role of curcumin combined with bone marrow transplantation in reversing experimental diabetes: Effects on pancreatic islet regeneration, oxidative stress and inflammatory cytokines. Eur J Pharmacol 658: 41-48, 2011. 